\title{
Agôn
}

Revue des arts de la scène

HS 1 | 2011

Mettre en scène l'événement

\section{Janvier 2011 / 14 January 2011}

Par les élèves de 1ère STG2 du Lycée VOILLAUME - Aulnay-sous-Bois

(Année scolaire 2010-2011)

\section{Collectif}

\section{(2) OpenEdition}

1 Journals

Édition électronique

URL : http://journals.openedition.org/agon/1802

DOI : 10.4000/agon.1802

ISSN : 1961-8581

Éditeur

Association Agôn

Édition imprimée

Pagination : $\mathrm{fr}$

\section{Référence électronique}

Collectif, « 14 Janvier 2011 / 14 January 2011 », Agôn [En ligne], HS 1 | 2011, mis en ligne le 02

septembre 2011, consulté le 17 avril 2020. URL : http://journals.openedition.org/agon/1802 ; DOI :

https://doi.org/10.4000/agon.1802 


\section{Janvier 2011 / 14 January 2011}

\section{Par les élèves de 1ère STG2 du Lycée VOILLAUME - Aulnay-sous-Bois (Année}

scolaire 2010-2011)

VOIX D'HOMME (Waël Ibrahim)

Je m’appelle Waël Ibrahim j'ai 19 ans

Je suis élève à l'Institut préparatoire aux études d'ingéniorat

VOIX DE FEMME (Ahlem Belhadj)

Mon nom est Ahlem Belhadj

J'ai 46 ans je suis pédopsychiatre et enseignante

À la faculté de médecine de Tunis

WAEL IBRAHIM

J'ai d'abord été militant blogueur à la fac

On ne se parlait que sur Internet

Mais jamais avec nos vrais noms

Par Facebook j'ai été informé

De la manifestation de vendredi 14 janvier 
AHLEM BELHADJ

Cette manifestation

Je ne l'aurais manquée pour rien au monde

WAEL IBRAHIM

J'y étais avec des amis

On s'est mis devant le ministère de l'intérieur

On criait Ben Ali Dégage

CHCEUR

From poor mothers with children

To denizens of the city's upper class

All singing the national

Anthem

Chanting Ben Ali Get Lost

VOIX D'UN AUTRE HOMME (Slim Amamou)

Je m’appelle Slim Amamou

J'étais prisonnier

AHLEM BELHADJ

J'étais là à $14 \mathrm{~h} 30$

SLIM AMAMOU

Hier le président a dit

AHLEM BELHADJ

Quand les flics ont chargé brusquement

On a couru 
SLIM AMAMOU

« On ne tirera plus sur la population »

WAEL IBRAHIM

Deux jeunes sont tombés devant moi

Tués de sang froid

Je ne pensais pas que les flics tireraient

\section{CHCEUR}

Suffering

Bloodshed

And lawlessness

JOURNALISTE

Treize manifestants ont été tués

Au cours de cette émeute dans la seule capitale

VOIX DE JEUNE HOMME (Ayoub)

Je m'appelle Ayoub

J'habite en Tunisie

Où je suis

Je n'ai assisté à aucune violence ni soulèvement

La ville a toujours été calme

\section{JOURNALISTE}

La ville de Tunis porte encore les stigmates de la violence

Carcasses de voitures neuves

AHLEM BELHADJ 
J'ai couru

J'ai foncé vers la rue de Paris

JOURNALISTE

Chars de l'armée dans les carrefours stratégiques

Hélicoptères en patrouille

AYOUB

Les seuls moments de liesse

Avec la foule ont eu lieu

Quand les affiches du président

Ont été enlevées et brûlées

CHCEUR

As news of the self-immolation spread

So did riots

JOURNALISTE

Les forces de l'ordre sont omniprésentes

WAEL IBRAHIM

En une fraction de seconde tout a basculé

Je me suis mis à courir

Et puis je me suis dit

BEN ALI

Je vous promets

VOIX DE JEUNE GARCON

C'est une pièce de théâtre 


\section{CHCEUR}

A growing population of young people

Who are at once

Educated and ambitious

BEN ALI

Plus de liberté

WAEL IBRAHIM

Que je n'allais pas passer ma vie à courir

VOIX D'HOMME (Mohamed Salah Fliss)

Mon nom est Mohamed Salah Fliss

J'ai 64 ans

Ancien prisonnier politique de l'époque Bourguiba

Longtemps militant du mouvement Perspectives

Et je n'en crois pas mes oreilles

SLIM AMAMOU

Hier le président a dit

WAEL IBRAHIM

Voilà la bouche qui nous a menti

BEN ALI

Je ne me représenterai pas aux prochaines élections

MOHAMED SALAH FLISS

Je n'ai pas bougé de chez moi

Télévision et ordinateur allumés téléphone 
À la main

SLIM AMAMOU

Il a donné des ordres

UN HOMME TRÈS ÂGÉ ET ÉMU

C'est le ministère de Tunis ici

J'ai travaillé au ministère de Tunis

\section{SLIM AMAMOU}

Où ils sont les ordres ?

L'HOMME TRÈS ÂGÉ ET ÉMU

Moi je remercie dieu

De m’avoir laissé assister à la liberté

J'ai vécu avec tous les régimes

L'ancien de Bourguiba et le reste

23 ans perdus comme ça

Et voilà

Voilà

Je suis arrivé à vivre une vraie liberté

Je sens la liberté maintenant

CHCEUR

We can hardly believe we're free

This is more than a dream

VOIX DE JEUNE GARCON

Avant personne n'osait parler 
CHCEUR

Unemployed and frustrated

BEN ALI

Plus de liberté

JEUNE GARCON

On était muselés

CHCEUR

Muzzled and resentful

SLIM AMAMOU

Hier le président a dit

LA FOULE

Ben Ali Dégage

BEN ALI

Je vous ai compris

JOURNALISTE

Des milliers ils étaient des milliers

À crier leur colère sur l'avenue Bourguiba

MOHAMED SALAH FLISS

Trente ans dans la vie d'un peuple

Ce n'est rien 
SLIM AMAMOU

Hier le président a dit

MOHAMED SALAH FLISS

Dans la vie d'un homme c'est beaucoup

BEN ALI

Je promets plus de pluralisme et de démocratie

CHCEUR

Resign

Resign

JOURNALISTE

13 manifestants ont été tués au cours de la nuit

BEN ALI

On ne tirera plus sur les manifestants à balles réelles

CHCEUR

Ben Ali destroyed many people

JOURNALISTE

Dans un discours télévisé hier soir

Le chef de l'État a annoncé plusieurs mesures d'ouverture politique

Pour calmer la rue

JEUNE GARCON

On n'a pas assez de mots pour dire notre colère 
JOURNALISTE

Dans le milieu de l'après-midi l'assaut a été donné contre la foule

Qui entourait le ministère de l'intérieur

\section{CHCEUR}

We proved in this country

That we're worthy of having a

Democracy

JEUNE GARCON

On ne va pas s'arrêter là

Ce qu'on ne veut pas

On le sait

SLIM AMAMOU

Les gens sont prêts à aller jusqu'à laisser leur vie

Pour que les choses changent

WAEL IBRAHIM

Pour l'instant

L'avenir est comme un grand brouillard

\section{CHCEUR}

I will always cherish the day the dictator Ben Ali was toppled

MOHAMED SALAH FLISS

Vendredi 14 janvier

Je me suis dit que l'avenir avait une chance

Une chance une chance une chance 
CHCEUR

I'll forever cherish 14 January 2011

Les élèves de 1ère STG2 du Lycée VOILLAUME - Aulnay-sous-Bois ayant pris part durant l'année scolaire 2010-2011 à l'écriture de cette pièce sont: Massim ASSOUMANE, Fatih AVSAR, Sabrina AITHOUMAD, Myriam BELLAHCEN, Safa BELMOKHTAR, Mouna BENMOUSSA, Laure CALLEEUW, Walid CHERMAK, Saliou DIALLO, Tahirou DIOMBERA, Foued ETTAOUFIQ, Eloise FEVRY, Anne-Sophie HOAREAU, Noémie ILE MABODY, Tarik ISSILI, Khalid KEMMOUS, Djénéba KONE, Ibrahim KOUMA, Maive LOUVILA, Anaïs MANOHARAN, Vishal MESSY, Karima MOUAOUED, Supitha NAVANEETHARAJAH, Stéphanie ROUSSEL, Ikram SADDAF, Anne-Sophie SALEZ, Soleine SANDJA, Franceline SATHIYASEELAN, Hakim SEFFAK, Moussou SISSOKO, Kiereddine SOUAG, Kandee SOUMARE, Silan TANRIVERDI, Soumail TRAORE, Elodie VONG.

\section{Pour citer ce document :}

«14 Janvier 2011 / 14 January 2011", Agôn [En ligne], Dossiers, D'un 11 septembre à l'autre, HS n 1 : Mettre en scène l'événement, mis à jour le : 05/09/2011, URL : http://agon.enslyon.fr/index.php?id=1802. 